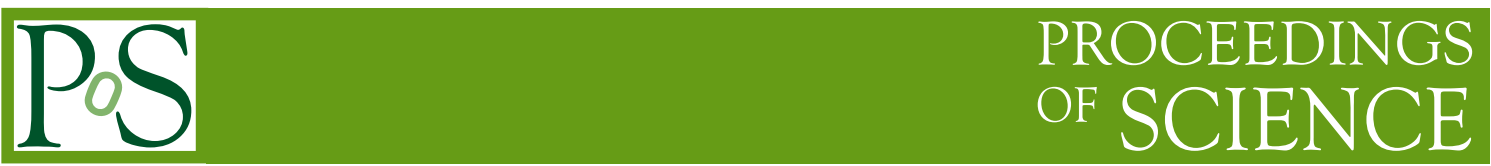

\title{
Astrophysical Jets: an updated, historical review
}

\author{
J. H. Beall* \\ Space Sciences Division, NRL, Washington, DC, USA \& St. John's College, \\ Annapolis, Maryland, USA \\ E-mail: beall@sjc.edu
}

We define astrophysical jets as linear structures detected in the sky, typically bi-polar, and originating from a common source. In the last three decades and more and as a result of observing campaigns using detectors sensitive from radio to $\gamma$-ray energies, theoretical models of these sources have become richer and more complex. Models have moved from assumptions of isotropy that made analytic calculations possible, to fully anisotropic models of emission from the jets and their interactions with the interstellar and intra-cluster medium. Such anisotropic calculations are only possible because we now have extensive computational resources that can solve the rather complex emission models that result from such anisotropies.

Recent observations by the neutrino detector system, ICECUBE, in concert with coordinated observations by the MAGIC Collaboration Cerenkov telescopes and the Fermi LAT, have for the first time given us direct evidence that the jets consist of hadronic elements. Remarkably, the recent observations of gold line emission produced by the kilonova associated with gravitational waves (see, e.g. Seigel, 2019) also suggest that jets form even during gravitational wave generation during compact NS-NS object coalescence.

keywords: astrophysical jets, active galactic nuclei, UHE cosmic rays, quasars, micro-quasars

Multifrequency Behavior of High Energy Cosmic Sources - XIII - MULTIF2019

3-8 June 2019

Palermo, Italy 


\section{Introduction}

Astrophysical jets have been defined as linear structures observed on the sky, sometimes bipolar, but sometimes seeming to be one-sided, and originating from a compact source. Jets are typically associated with outflows originating from accretion processes in star-forming regions and in close binary systems containing compact objects (i.e., white dwarfs, neutron stars, and black holes), and of course in active galactic nuclei (AGNs), and galaxy clusters. They also appear to be present in $\gamma$-ray bursts associated with stellar collapse when the jet is pointed at the Earth, and in some pulsars. In pulsars, the required energy for jet production can be drawn from angular momentum loss of the pulsar. In accreting systems, the jet production seems to be entangled intimately with processes in the accretion disks around the compact objects.

Astrophysical jets can be extremely powerful in terms both of their kinetic luminosity and radiative signatures, and often emit radiation over the entire electromagnetic spectrum from radio to $\gamma$-rays. The jets often appear to be one-sided, or to have asymmetric brightness in one of the "lobes." This phenomenon is usually interpreted as evidence of bulk relativistic motion of the emitting particles which produces a Doppler boosting of the approaching lobe's brightness, and a dimunition of the brightness of the jet lobe receding from the observer.

Early papers by Beall et al. (1978), and Beall and Rose (1980) have noted that the luminosity of the central source in Cen A, which is clearly connected to the giant radio lobes via the jets flowing outward from the core of that galaxy, could provide the energy to power the giant radio lobes.

The results of observational campaigns show both similarities and significant differences in the data from some epochs of galactic microquasars, including GRS 1915+105 (Mirabel, et al., 1998; Rahoui et al., 2008), the concurrent radio and x-ray data (Beall et al., 1978) on Centaurus A (NGC 5128), 3C120 (Marscher (2006), and 3C454.3, as reported by Bonning et al. (2009), and Cygnus A (Carilli, et al., 1996), to name a few efforts. The Bonning et al. analysis of the 3C454.3 data showed the first results from the Fermi LAT Space Telescope (Abdo 2009a, 2009b) for the concurrent variability at optical, UV, IR, and $\gamma$-ray frequencies for that source.

In combination with observations of microquasars and quasars from the MOJAVE Collaboration (see, e.g., Lister et al., 2009), these efforts provide an understanding of the time-dependent evolution of these sources at milliarcsecond resolutions (i.e., parsec scales for AGNs, and Astronomical Unit scales for microquasars).

Integrated models of AGN-jet systems (see, e.g., Urry and Padovani, 1995) have greatly helped our understanding of the different guises in which jet-ambient medium interactions can make their appearances.

The recent possibility of observational advances via millimeter wave interferometers has the potential of allowing us to the remarkable variability and structure of these objects on even finer scales.

Because of these campaigns both past and present, models of jet sources have progressed from earlier assumptions of isotropy that made analytic calculations possible, to fully anisotropic models of emission from the jets and their interactions with the interstellar and intra-cluster mediums. Furthermore, observations of the temporal behavior on milliarcsecond scales have allowed us to understand that the source regions are not simple, but have a complex and evolving structure. 
In large measure, such models of anisotropic structures and temporal variability are only possible because we now have extensive computational resources to model the jets' structures. In addition, the degree of international cooperation required for observing campaigns of these sorts is remarkable, since the instruments include among others the Very Large Array (VLA), the Very Long Baseline Array (VLBA), and entire constellations of satellite instruments, often working in concert. In this paper, I discuss some relevant observations from these efforts and the theoretical interpretations they have occasioned, with some emphasis on the history of our evolving understanding of the astrophysical jets.

In blazar sources (see, e.g., Ulrich et al. 1997, and Marscher, 2006) there seems to be a confirmed connection of jets with accretion disks. In sources without large-scale linear structures (i.e., jets), as Ulrich et al. (1997) note, the source variability could result from the complex interactions of the accretion disk with an x-ray emitting corona, and perhaps with the gravitational potential of the disks, themselves. But to the extent that "small" jets are present in these sources, the disk-jet interaction must still be of paramount importance, since it provides a mechanism for carrying away energy from the disk.

Current theories suppose that the jet is formed and accelerated in the inner regions of the accretion disk (see e.g., Hawley 2003 and Bisnovatyi-Kogan, et al., 2013 for a discussion of disk structure and jet-launch issues, respectively). But even if this is true in all sources, it is still unclear whether or not astrophysical jets with shorter propagation lengths are essentially different in constitution from those that have much longer ranges, or whether the material through with the jet propagates determines the extent of the observational structures we call jets. At all events, the complexities of the jet-ambient-medium interaction must have a great deal to do with the ultimate size of emitting region and the energy deposition scales.

This sort of argument has applications to both quasars and microquasars, especially if essentially similar physical processes occurs in all these objects (see, e.g., Beall, 2003, and Marscher, 2006). To some, it has become plausible that essentially the same physics is working over a broad range of temporal, spatial, and luminosity scales. Hannikainen (2008) and Chaty $(1998,2007)$ have discussed some of the emission characteristics of microquasars, and Paredes (2007) has considered the role of microquasars and AGNs as sources of high energy $\gamma$-ray emission. In fact, the early reports of the concurrent radio and x-ray variability of Centaurus A can be plausibly interpreted as the launch of a jet from Cen A's central source into the complex structures in its core. Additionally, these observations are remarkably similar to the observations of galactic microquasars and other AGNs, including the observations from the Fermi LAT Space Telescope of concurrent $\gamma$-ray, IR, optical, and UV variability of 3C454.3 (Bonning et al., 2009), and observations by Madejski et al. (1997) for BL Lac, among others.

\section{Jets from Accretion Disks in Star-Forming Regions}

There is evidence for accretion disks associated with the bipolar outflows in star-forming regions such as the rho Ophiuchus cloud (see, e.g., Beall 1987, and the rho Ophiuchus jet observations taken from K. Tachihara's Ph.D. thesis). The data therein interpreted show that the IR spectra in the centers of the regions of bi-polar flows are consistent with the emission expected from accretion disks with the thermal gradient expected from a Shakura-Sunyaev (1973) accretion disk. 
The spectrum calculated by Beall (op. cit.) agrees with the illustration presented by Hannikainen (2005).

Recent observations bear out this hypothesis. For example, the observations of HL Tauri with the Atacama Large Millimeter/Sub-Millimeter Array (ALMA) presented in Figure 1 clearly show the resolved accretion disk structure of the protostellar system beginning to segregate itself into rings that are hypothesized to become proto-planets. The observations from ALMA are also corroborated by data from Owens Valley Radio Observatory's Combined Array for Research in Milimeter-Wave Astronomy (CARMA), as reported by Stephens et al. (2014). In addition, and perhaps most importantly, the CARMA date show a magnetic field morphology of at 0.6 arcsecond resolution. The polarization vectors appear to show a magnetic field perpendicular to the disk orientation.

More recently, observations by ALMA (see, e.g. Lee 2017, and Figure 2) show a classical jet-structure along with a rotating accretion disk! The innermost part of the jet in $\mathrm{SiO}$ within approximately $120 \mathrm{AU}$ of the central source with a resolution of roughly $8 \mathrm{AU}$ on top of the continuum map of the disk. See Pudritz and Ray's 2019 review for a detailed discussion, and Lee et al. (2017) for the observational report.

Remarkably, this resolved accretion disk is clearly associated with a jet structure (Rodriguez, et al., 1994) for data taken by the VLA on arcsecond scales.

The observations shown in Figure 2, taken from Pudritz and Ray's (2019) review and originating with Lee et al.'s observations (2017), represent the innermost part of the jet in $\mathrm{SiO}$ lines within roughly $120 \mathrm{AU}$ of the central source, at a resolution of approximately $8 \mathrm{AU}$ on top of the continuum map of the disk. The intensity (in units of $\mathrm{K} \mathrm{km} / \mathrm{s}$ ) integrated over certain velocity range shows the quite interesting features of the knot-like structure that manifests itself in these sources as well as AGN systems and microquasars (to be discussed in the following sections). This phenomena must be tied to the jet-launch process in the accretion disk. Most importantly, the jet structure is clearly seen to be rotating, and there is evidence in the details of extended magnetic fields associated with the jet launch and propagation.

\section{Jets in Microquasars}

The usual story about micro-quasars is that they are miniatures of their more blatant cousins, the Active Galactic Nuclei. This is true in gloss, but bears some investigation. I remind the reader of the now-famous Mirabel micro-quasar, GRS 1915+105 (see, e.g., Mirabel,1994, and Dahwan et al., 2006 for reviews) which demonstrated that the canonical blob-like structure of the astrophysical jets for material ejected from the cores of AGN was a commonplace in jets associated with binary black-hole sources in our galaxy and others.

Furthermore, the quite interesting story of the jet in Sco X-1, a neutron star binary system, shows that jet ejection shows no favorites with respect to gravitational sources, since it not only occurs in the formation of ordinary stars, but also in accretion onto black-holes and neutron stars.

Remarkably, the recent kilonova detection of a jet-like structure associated with gravitational wave generation (discussed later in this review) shows that such effects are commonplace in those circumstances, as well. 


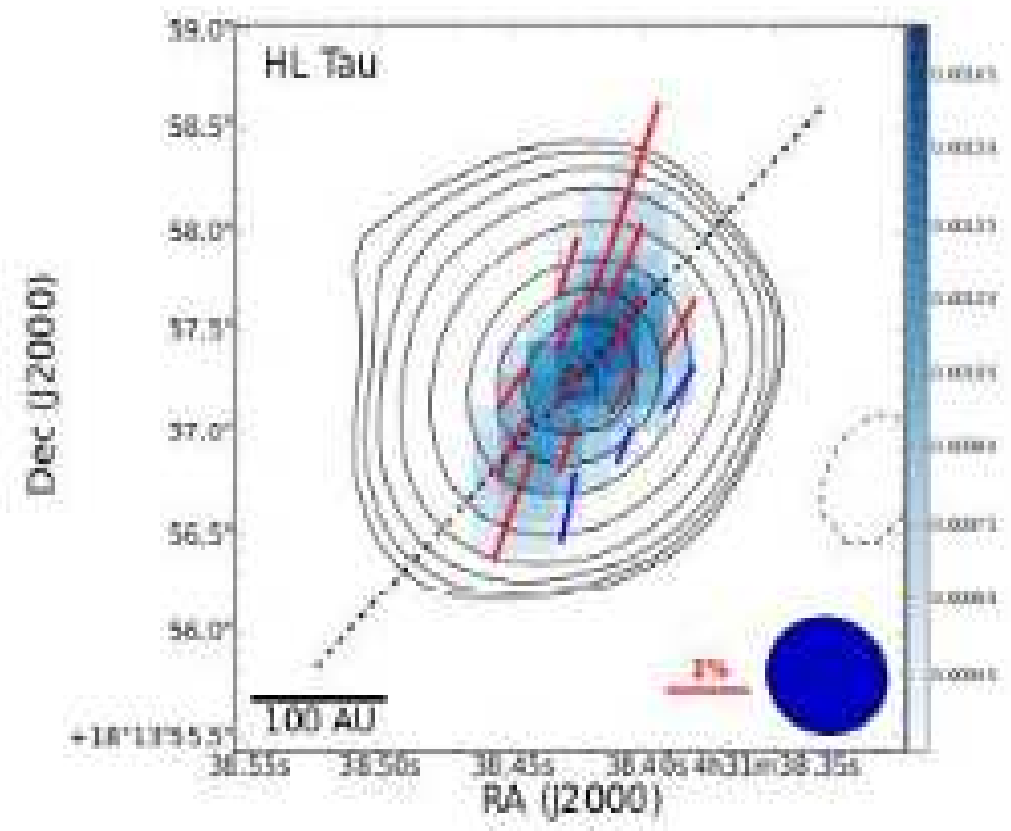

Figure 1a

\section{Figure 1b}

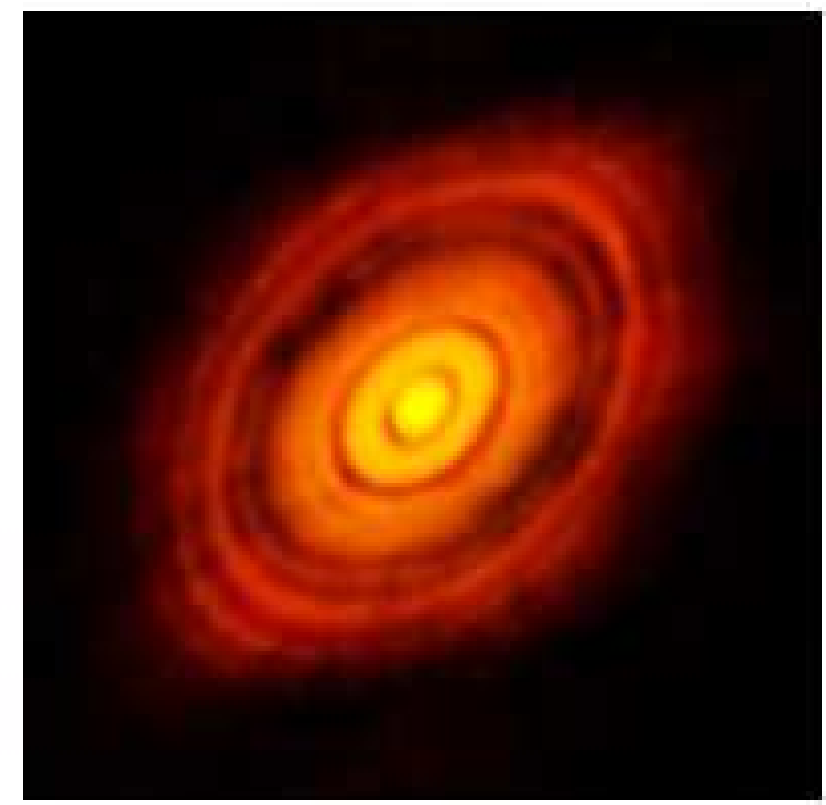

Figure 1: HL Tauri Figure 1a: Radio polarization data from the CARMA (Combined Array for Research in Millimeter-wave Astronomy) observatory show magnetic structures consistent with a jet perpendicular to the disk plane (Stephens et al, 2014). Figure 1b: Maurita image from ALMA: Note the ring-like structure that is likely to represent the beginnings of planetary formation in the protostellar system. This image is from ALMA (Atacama Large Millimeter/Sub-Millimeter Array and is on the same scale as the CARMA data (see, e.g., http://apod.nasa.gov/apod/ap141110.html). 

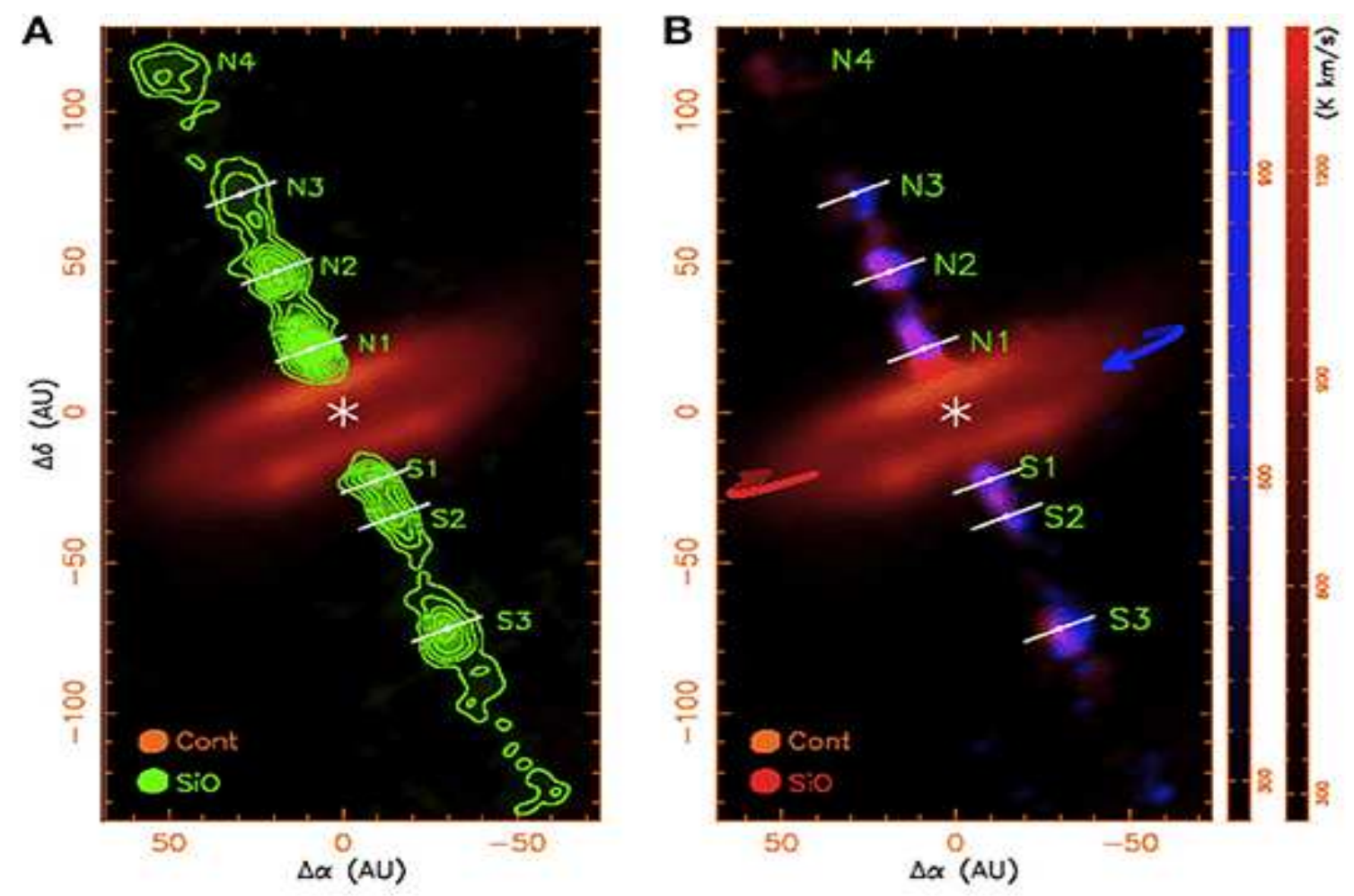

Figure 2: ALMA observations of the rotating outflows of the class 0 protostar HH 212 are shown in the image above. In this image, taken from Pudritz and Ray's 2019 review and originating with Lee et al.'s observations (2017), the innermost part of the jet in $\mathrm{SiO}$ lines is within $120 \mathrm{AU}$ of the central source, at a resolution of roughly $8 \mathrm{AU}$ on top of the continuum map of the disk. The maps show the intensity (in units of $\mathrm{K} \mathrm{km} / \mathrm{s}$ ) integrated over certain velocity range. It is quite interesting that the knot-like structure manifests itself in these sources as well as AGN systems and microquasars (to be discussed in the following sections). This phenomena must be tied to the jet-launch process in the accretion disk. Most importantly, the jet structures clearly seem to be rotating, and there is evidence in the details of extended magnetic fields associated with the jet launch and propagation.

But no historical review of microquasars could be complete without acknowledging the contribution of Fomalont, Geldzahler, and Bradshaw's work to uncover the jet emission from the binary neutron star, Sco X-1. As they tell it, the hints of extended GHz radio emission from Sco X-1 were both tantalizing and evanescent. Over several years (Fomalont, Geldzahler, and Bradshaw chased an elusive phantom, finally "nailing it" as they say with a week long observational campaign that showed not only the jet evolving from the flare at the central source, but also a jet with both slowmoving (though still-relativistic) blobs, and faster "bullets" whose existence they inferred. Even more interesting is that the asymmetric brightness structure of the jets and their associated timedelay are beautiful examples of relativistic Doppler boosting and relativistic time delays (see.e.g., Bradshaw, Geldzahler, and Fomalont (1999) and Fomalont, Geldzahler, and Bradshaw, (2001)).

It is also of interest that the consideration of SS 433 as a galactic microquasar and the observations of its extended magnetic field structures (see Waisberg, I., Dexter, J., Olivier-Petrucci, P., Dubus, G., and Perraut, K. 2019 for a review of its history) suggest that we consider extended 
magnetic field structures in other sources as well, including even AGNs and Quasars.

We now turn to jets associated with AGNs and quasars.

\section{Concurrent Variability of AGN Jet Sources as an Indication of Jet Launch or Jet-Cloud Interactions}

As examples, we now turn to two such observations in this paper, the concurrent radio and X-ray variability of Centaurus A (Beall et al., 1978), and the $\gamma$-ray, UV, and IR concurrent variability discussed by Bonning et al., (2009) using the Fermi LAT and Swift spacecraft.

\subsection{Radio and X-ray Variability Measurements of Centaurus A (NGC 5128)}

The first detection of concurrent, multifrequency variability of an AGN came from observations of Centaurus A (Beall et al., 1978, see Figure 1 of that paper). Beall et al. conducted the observing campaign of $\mathrm{Cen} \mathrm{A}$ at three different radio frequencies in conjunction with observations from two different $\mathrm{x}$-ray instruments on the OSO-8 spacecraft in the $2-6 \mathrm{keV}$ and $100 \mathrm{keV}$ x-ray ranges. These data were obtained over a period of a few weeks, with the Stanford Interferometer at $10.7 \mathrm{GHz}$ obtaining the most data. Beall et al. also used data from other epochs to construct a decade-long radio and x-ray light curve of the source. Figure 1a of Beall (2011) shows the radio data during the interval of the OSO-8 x-ray observations, as well as the much longer timescale flaring behavior evident in the three different radio frequencies and at both low-energy (2-6 keV, see Figure $1 \mathrm{~b}$ of Beall, 2011) and in high-energy ( $100 \mathrm{keV}$, see Figure 1c of Beall, 2011) x-rays. As noted by Beall (2011), a perusal of Figure 1a in that paper shows that the radio data (represented as a "+" in that figure) generally rise during 1973 to reach a peak in mid-1974, then decline to a relative minimum in mid-1975, only to go through a second peak toward the end of 1975, and a subsequent decline toward the end of 1976. This pattern of behavior is also shown in the $\sim 30 \mathrm{GHz}$ data and the $\sim 90$ $\mathrm{GHz}$ data albeit with less coverage at the higher two radio frequencies. These data are discussed in more detail in Beall (2015).

In retrospect, a plausible hypothesis for the Cen A observations is that they arise from physical processes associated with the "launch" of an astrophysical jet into the complex structures in the core of Centaurus A. The timescale of the evolution from early 1973 through 1977 appears to be associated with the evolution of a larger structure over a more extended region. Again, the observations are consistent with the interaction of an astrophysical jet with an interstellar cloud in the core of Cen A.

Recent work on modeling such an interaction of jets with "clumpy" interstellar media and interstellar clouds, (see, e.g., Perucho and Bosch-Ramon et al., 2011, and Silk, et al., 2012),

It is clear from this discussion that a distinction needs to be made about which observational signatures are associated with the jet launch, the jet itself, and the ambient medium's reaction to the jet. In considering such a scenario applied to microquasars, the observations of Sco X-1 by Fomalont, Geldzahler, and Bradshaw (2001), as discussed above, are extremely informative. Such patterns of flaring have also been observed in other AGNs, including Blazars. 


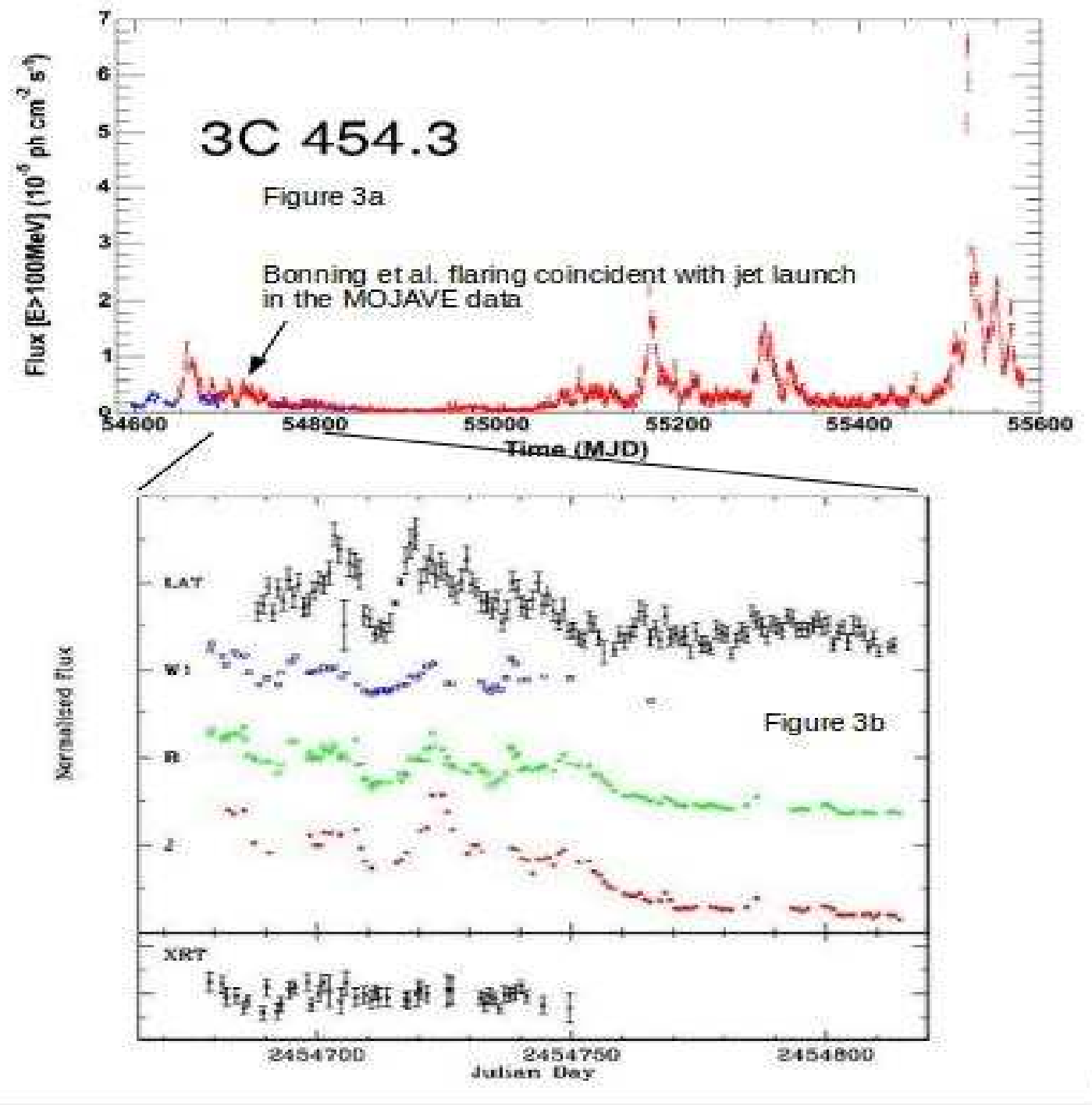

Figure 3: 3C454.3 data taken from Fermi LAT data (see, e.g., Lott et al. 2013) showing the time history of the flaring from $3 \mathrm{C} 454.3$ along with times for several additional flares. The lower panel shows the variability of 3C 454.3 Fermi 0.1-300 GeV gamma-ray, UV (W1), optical (B), and IR (J), and X-ray (XRT), taken from Figure 1 of Bonning et al. 2009, ApJ Letters 697, L81 (and ArXive:0812.4582v1). The flaring in the MOJAVE data is also indicated by the black arrow in the upper panel, and is coincident with the flares in the Bonning et al., data. and clearly indicate the correlation of $\gamma$-ray flares with the jet launches. These associations were first noted by Beall (2012) and have been have also been analyzed extensively by Jorstadt et al. (2013). In total, the data presented here show four concurrent $\gamma$-ray and radio flares associated with the launch of material that comprises an astrophysical jet. 


\subsection{A Jet Launch Coincident with the 3C454.3 Multi-frequency Flaring}

Bonning et al. (2009) performed an analysis of the multi-wavelength data from the blazar, 3C454.3, (see Figure 3) using IR and optical observations from the SMARTS telescopes, optical, UV and X-ray data from the Swift satellite, and public-release $\gamma$-ray data from the Fermi LAT experiment. In that work, she demonstrated an excellent correlation between the IR, optical, UV and $\gamma$-ray light curves, with a time lag of less than one day.

Urry (2011) noted that 3C454.3 can be a laboratory for multifrequency variability in Blazars, and this has indeed, become true. Beall (2016) first noted that the successive flares from the MOJAVE data were coincident with Fermi LAT flaring, and that the characteristic signatures of the subsequent flares (see Figure 03) showed remarkably similar patterns to that discussed in Bonning et al. (2009)

A more extensive analysis performed by Jorstadt et al. (2017) affirms the very tight correlations between MOJAVE data and the milliarcsecond jet launches and the FERMI LAT flaring. Continued monitoring of $3 \mathrm{C} 454.3$ will be helpful to refine models of AGN sources. But it is clear from the similarity between observed concurrent flaring at the different frequencies, that the pattern of a correlation between low-energy and higher-energy variability is consistent with that observed for Cen A, albeit with the proviso that the energetics of the radiating particles in $3 \mathrm{C} 454.3$ is considerably greater. Most importantly, a perusal of the data for 3C454.3 at milliarcsecond scales taken from the MOJAVE VLBA campaign during the Bonning et al (2009) flare and the subsequent flares show that the time-dependent flares occur during launch of new components of the jet that originates from the core.

It should be emphasized that other epochs in the 3C454.3 data from MOJAVE and Fermi LAT also show similar periods of injection of radio blobs that are associated with a double-peaked structure in the $\gamma$-ray light. The Fermi LAT data (see, e.g., Lott et al. 2013) show that the time history of the flaring from 3C454.3 are associated with times for several additional flares in the MOJAVE data sets (see Figure 3). The flares are shown in Figure 3, and clearly indicate the correlation of $\gamma$-ray flares with the jet launches. These associations have also been pointed out by Jorstadt et al. 2013, who provide a detailed review of three outbursts in their paper. The direct correlation of the Fermi LAT and the MOJAVE data can clearly be seen in the figure. The data show evidence for the repeated launching of the astrophysical jets into the ambient medium. The jet structure is apparently not a continuous injection over long time scales, but an injection of a series of "blobs" that are co-aligned along some preferred axis.

This last statement cannot, however, be used as an argument for simple, spherically symmetric models of these sources. This is especially true since the injected material (itself unlikely to be symmetric by any definitions of the word) immediately interacts with highly asymmetric magnetic fields and structures in the accelerating region and in the ambient medium in the source's core. The result of these phenomena is a dynamic, highly anisotropic emitting region that produces anisotropic radiation over the entire electromagnetic spectrum.

For the Cen A data, as for data from 3C454.3, it was the concurrent variability that suggests that the radio to x-ray (in Cen A's case) and the IR, optical, and UV to $\gamma$-ray fluxes (in 3C454.3's case) are created in the same region. This leads to the possibility of estimates of the source parameters that are obtained from models of these sources. VLBI observations of cores vs. jets (see, e.g., the 


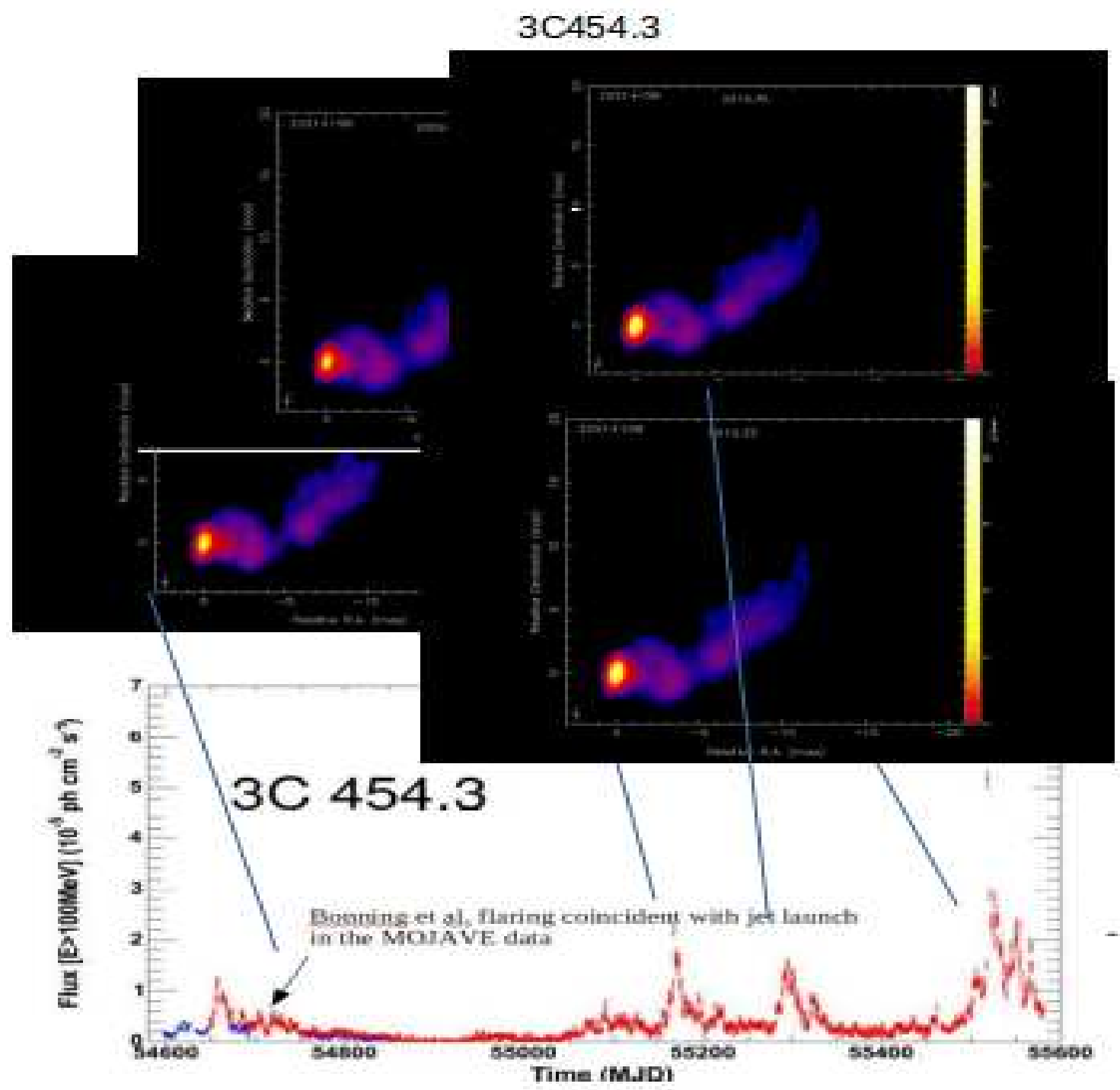

Figure 4: Fermi LAT data (see, e.g., Lott et al. 2013) showing the time history of the flaring from 3C454.3 along with times for several additional flares in the MOJAVE data sets, The flares are similar to those shown in Figure 3 at later times, and clearly indicate the correlation of $\gamma$-ray flares with the jet launches. These associations have also been analyzed extensively by Jorstadt et al. (2013), who provide a detailed review of the outbursts in their paper. The direct correlation of the Fermi LAT and the MOJAVE data can clearly be seen in the figure. The three additional flares are similar in temporal structure to those illustrated in Figure 4. In total, the data presented here show four concurrent $\gamma$-ray and radio flares associated with the launch of material that comprises an astrophysical jet. 
study of BL Lac by Bach et al. 2006) show the structures of the core vs. jet as they change in frequency and time. It has thus become possible to separate and study the time variability of the jet and the core of AGN at remarkably fine temporal and spatial scales.

Van der Laan (1966) has discussed the theoretical interpretation of cosmic radio data by assuming a source which contained uniform magnetic field, suffused with an isotropic distribution of relativistic electrons. The source, as it expanded, caused an evolution of the radio light curve at different frequencies. Each of the curves in Van der Laan's paper represents a factor of 2 difference in frequency, the vertical axis representing intensity of the radio flux and the horizontal axis representing an expansion timescale for the emitting region. Van der Laan's calculations show a marked difference between the peaks at various frequencies.

In the example of Cen A, the radio frequency bands track one another throughout the entire interval reported by Beall et al. (1978). The data from Cen A (as discussed more fully in Beall $(2008,2010)$ are, therefore, not consistent with van der Laan expansion (Van der Laan, 1966), since for van der Laan expansion, we would expect the different frequencies to achieve their maxima at different times. Even if the $10.7 \mathrm{GHz}$ data are in the synchrotron self-absorbed portion of the spectrum, as appears likely, there is no straightforward way to explain the behavior of the radio data via the van der Laan mechanism.

The observations in the hard $\mathrm{x}$-ray at $100 \mathrm{keV}$ are also problematic, since the slope of the power-law spectrum at $100 \mathrm{keV}$ hardens (i.e., flattens) at one epoch in the observations. The hard $\mathrm{x}$-ray spectrum is unabsorbed, and therefore represents the spectrum of the underlying radiating electrons. Therefore, the most likely explanation for the changes in the spectrum of the Cen A data at $100 \mathrm{keV}$ (Beall, et al., 1978) is that the emitting region suffered an injection or reacceleration of energetic electrons.

A similar scenario can explain the reported radio variability. That is, the jet-ambient-medium interaction injected a population of energetic particles into a putative van der Laan "blob," or, equivalently, that there was a re-acceleration of the emitting electrons on a timescale short compared to the expansion time of the source.

An analysis of the $3 \mathrm{C} 120$ results compared with the data from the galactic microquasar, Sco X-1, undertaken by Beall (2006) shows a similar radio evolution, with rapidly moving "bullets" interacting with slower moving, expanding blobs. It is highly likely that the elements of these sources that are consistent with van der Laan expansion are the slower-moving, expanding blobs. I believe that the relativistically moving bullets, when they interact with these slower-moving blobs, are the genesis of the flaring that we see that seems like a re-acceleration of the emitting, relativistic particles. I note that a similar scenario could be operating in Cen A and 3C454.3.

This is not to say that the "slower-moving blobs" are not themselves moving relativistically. It is clear that the bi-polar lobes have significant enhancements in brightness due to relativistic Doppler boosting for the blobs moving toward us.

The true test of this hypothesis will require concurrent, multifrequency observations with resolutions sufficient to distinguish jet components from core emissions in galactic microquasars as well as for AGN jets.

To reiterate from the previous section on microquasars, one of the most remarkable sagas regarding the discovery of quasar-like activity in galactic sources comes from the decades longinvestigation of Sco X-1 by Ed Fomalont, Barry Geldzahler, and Charlie Bradshaw (Fomalont, 
Geldzahler, and Bradshaw, et al. 2001). During their observations, an extended source changed relative position with respect to the primary object, disappeared, and then reappeared many times. We now know that they were observing a highly variable jet from a binary, neutron star system. The determinant observation was conducted using the Very Large Array (VLA) in Socorro, New Mexico and the VLBA interferometer (see, e.g., Beall, 2008) for a more complete discussion).

It is worthy of note that the milliarcsecond observations show a complex evolution of structure at parsec scales, including an apparently sharp change of directions associated with changes in the polarization of the radio light at that point in the jet's evolution. Furthermore, the multi-frequency flares reported by Bonning et al.(2009) are consistent with the launch of another component of the astrophysical jet in the core region.

Regarding the acceleration region and the possible mechanisms for the collimation of the jets, a number of models have been proposed (see, e.g., Kundt and Gopal-Krishna (2004), BisnovatyiKogan et al. (2002), Romanova and Lovelace (2009), and Bisnovatyi-Kogan, et al. (2013) that might help explain the complexity present in these data.

The observations of the concurrent IR, Optical, UV, and $\gamma$-ray variability of $3 \mathrm{C} 454.3$, and its associated jet launch in the MOJAVE data, argue for a continued monitoring of this source.

\section{Microarcsecond Measurements of Astrophysical Jets at Millimeter Wavelengths}

The milliarcsecond observations of 3C454.3 by MOJAVE show a complex evolution of structure at parsec scales, including an apparently sharp change of directions associated with changes in the polarization of the radio light at that point in the jet's evolution. Furthermore, the multi-frequency flares reported by Bonning et al. (2009) are consistent with the launch of another component of the astrophysical jet in the core region.

In trying to connect the arcsecond scale behavior of astrophysical jets to scales of milliarcseconds, we are naturally lead to the question of the behavior of the jet at microarcsecond scales.

The recent development of millimeter-wave interferometry, including the opening of the Event Horizon Telescope (or EHT, see e.g. Doelman et al. 2019) provides an ability to look "down into" the cores of AGNs. As noted by other authors, the ALMA is fortunately configured for Southern Sky Sources. Recent observations of M87 by the EHT show a remarkable structure to the region near the supermassive black hole, even to the point of allowing us to suppose that the EHT observations show the time-dependent structure of the accretion disk.

The data in Figure 5 show the results of the The Millimeter-Wave Interferometer (a.k.a., the Event Horizon Telescope) Collaboration taken in 2017 and published by Kim et al. (2019). The ring of light shown in the image is supposed to be produced by plasma that is swirling around the black hole. Hypothetically, this should represent some aspect of the accretion disk around M87's supermassive black hole. In fact, Tamburini, Thide, and Della Valle (2019) have used these data to argue for a determination both the orientation and rotation rate of the accretion disk that generated the M87 astrophysical jet.

According to Jeffries (2019) the M87 supermassive black hole "is likely to be spinning and its spin-axis is likely to be aligned with the large scale "jet" that is seen to emanate from the core of M87". They suppose that the direction of that jet is more-or-less E-W in the picture, and approaching 


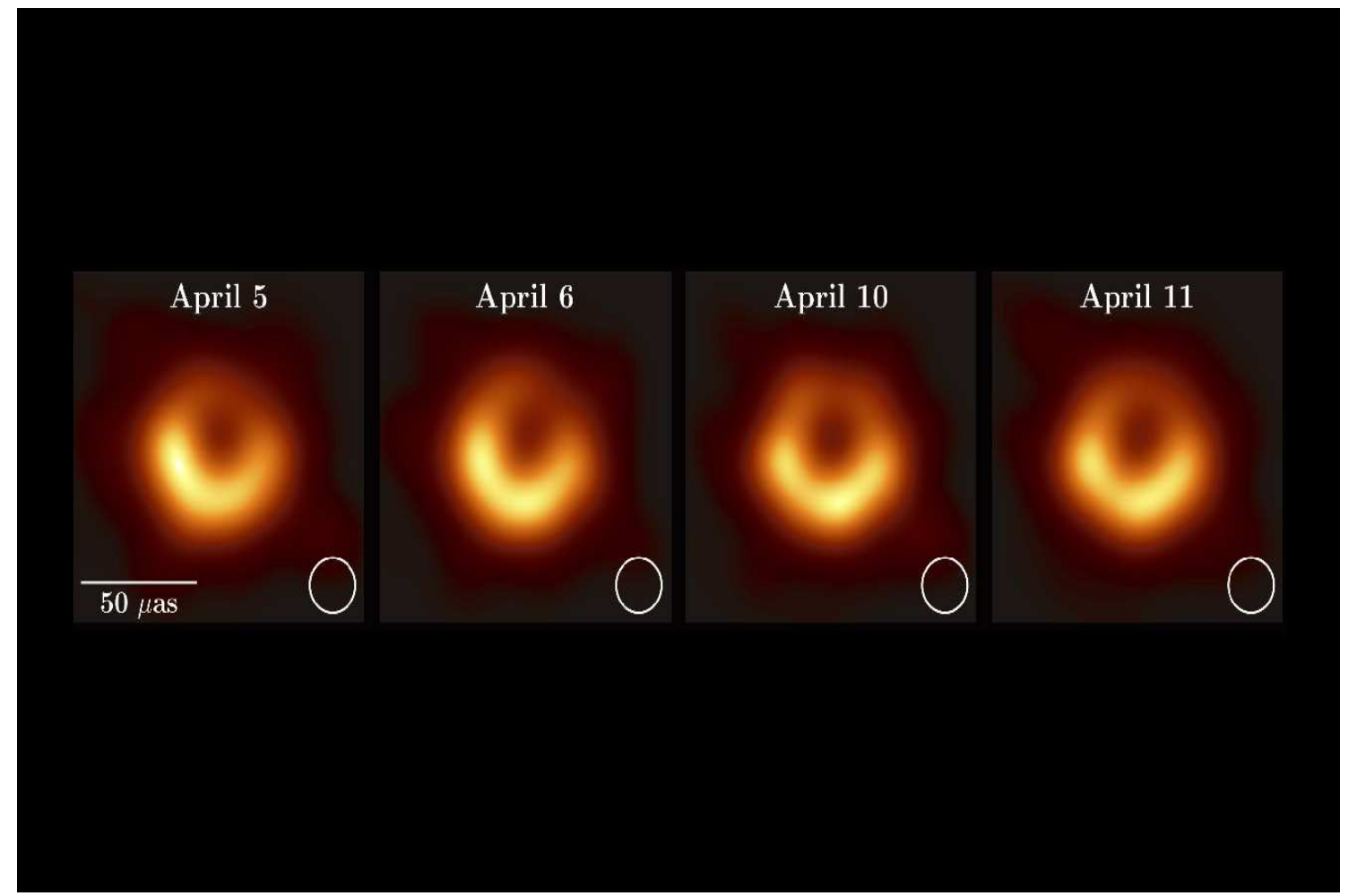

Figure 5: The Millimeter-Wave Interferometer (a.k.a., the Event Horizon Telescope) Collaboration. See, e.g., Kim et al., (2019), Doeleman et al., (2019), which show the time evolution of the millimeter wave signature of the ring of plasma around the massive black hole in M87

the observer at at an angle of roughly 17 degrees between the jet axis and our line of sight. This analysis is corroborated by Tamburini et al. (2019)

According to those authors, the microwave emission at $1.3 \mathrm{~mm}$ wavelengths is bent by the extreme gravitational field so that the light rays observed are gravitationally lensed. This suggests that the ring of light is not the accretion disk, but a magnified halo of light caused by gravitational lensing of the general illumination surrounding the black hole.

This scenario seems plausible, since it is likely that the black hole has significant angular momentum in order to produce the jet in the first place.

The black hole is therefore likely to be spinning either almost clockwise or anti-clockwise as we view it from Earth. The spin of the black hole means that plasma falling into it will be dragged in the direction of rotation at relativistic speeds. As it does so, any light emitted will be Doppler boosted in the direction that the material is moving.

The provisional analysis of Jeffries suggesting the 17 degree tilt from our line of sight means that there is a component of the velocity of the material circulating around the black hole that is either towards or away from us. They suggest that the brighter light from the south of the supposed disk in the picture means that material circulating around the black hole is coming towards us from south of the black hole. The spin-axis of the black hole must, according to Jeffries' analysis, be aligned with the jet. Again, it's projected spin-axis is roughly E-W on the picture) but pointing into 

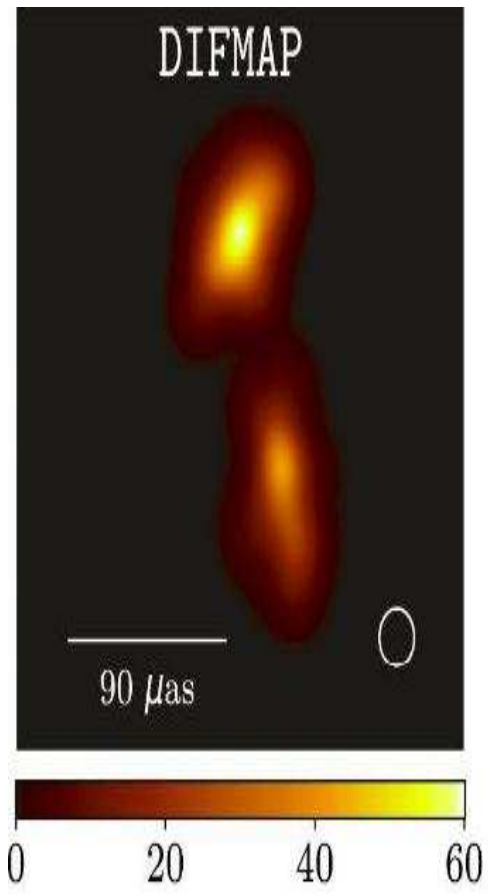

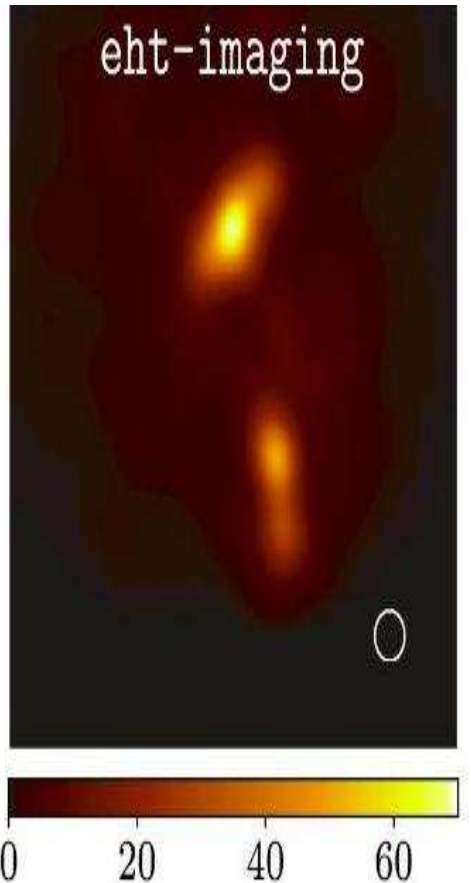

60

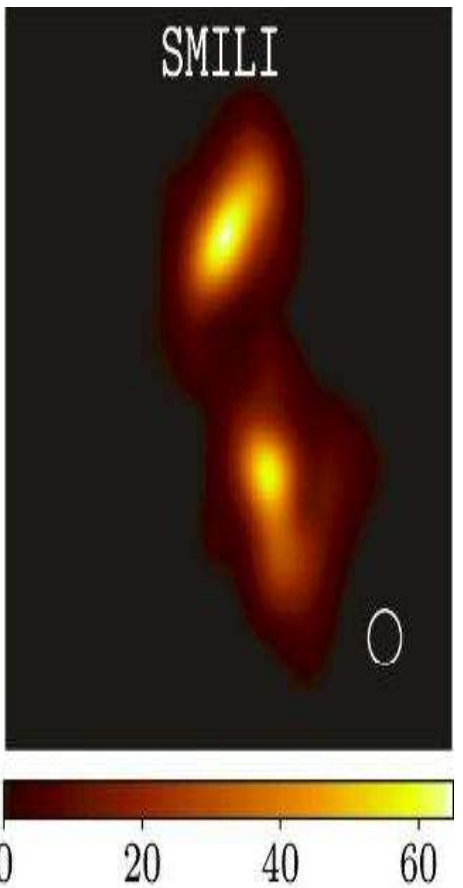

Brightness Temperature $\left(10^{9} \mathrm{~K}\right)$

Figure 6: Figure 6 shows representative images of 3C279 from the April 112019 EHT observations (see, e.g. Kim et al., 2019, Doeleman et al., 2019) produced using DIFMAP, EHT-imaging, and SMILI. To simplify visual comparisons and display the images at similar resolutions, the images are restored with circular Gaussian beams of 20, 17.1, and 18.6 microarcseconds FWHM, respectively. The observations were made in 2017; the image was published in 2019.

the picture (in a right-hand-rule sense), with most of the spin then being characterized as clockwise rotation. Again, $i$ this scenario, the jet is aligned with the spin axis of the supermassive black home.

Curiously, as we have seen from observations of other sources (such as Bonning et al.'s analysis of 3C454.3 shown above), at milliarcsecond scales the jets can diverge wildly from the alignments supposed by the arcsecond scale structures. We would then expect that some aspect of the spin axis of the supermassive black hole and its accretion disk actively aligns the astrophysical jet on scales of hundreds to thousands of parsecs, while at the same time allowing for jet precession on parsec and sub-parsec scales.

Recently, the Millimeter Wave Interferometer System (the EHT) has published initial observation of 3C279 (Figure 6). The panel shows data from 11 April 2017 at a wavelength of $1.3 \mathrm{~mm}$. The panel shows remarkable observations of scales from tens of microarcseconds and hundreds of arcseconds (see Kim et al, 2020). The finest resolution shows believable structure for the source at scales of tens of microarcseconds.

These observations suggest that the structure of the jets at scales down of microarcseconds maintains the pattern we see in other sources at millarcseconds, and thus directly links the "stable" arcsecond jets to the accretion disks around black holes. 
Said another way, it appears the direction of propagation of the jets changes radically on scales down to the limit of the accretion disk around massive black holes in active galactic nuclei, but manages to become stable on arcsecond scales. Therefore, we can link the milliarcsecond and microarcsecond structure of the astrophysical jets down to that scale.

From this linkage, a natural question arises: what is the mechanism for such a remarkable alignment? One can suppose that the most likely mechanism on scales ranging from the size of the accretion disk to kiloparsecs is a magnetic field, highly dynamic near the accretion disk from whence it is generated, and evolving outward to larger and larger scales to the point where it orders the arcsecond scale jets. In the process of interacting with the ejecta that arise from the recombination of intense magnetic fields in the inner region of the AGN black hole accretion disk, the magnetic field must be transformed from its relatively turbulent origins to a much more ordered system.

Of course, this hypothesis bears further investigation, but the most likely mechanism for such alignments are magnetic field structures arising from near the black hole event horizon and continuing outward to scales of at least hundreds of parsecs. The energetics of such a system of magnetic fields must be remarkable, and its source should be a topic of current research, both observationally and via modeling. Again, the images of 3C279 from the April 11 EHT observations produced using DIFMAP, eht-imaging, and SMILI show remarkable structure. To simplify visual comparisons and display the images at similar resolutions, those authors have shown the images restored with circular Gaussian beams of 20, 17.1, and 18.6 microarcseconds FWHM, respectively. These observations were made in 2017; with the image published in 2019 (see Kim et al., 2020, for a more detailed analysis)

Regarding the acceleration region and the possible mechanisms for the collimation of the jets, a number of models have been proposed (see, e.g., Kundt and Gopal-Krishna (2004), BisnovatyiKogan et al. (2002), Romanova and Lovelace (2009), and Bisnovatyi-Kogan, et al. (2013) that might help explain the complexity present in these data. But clearly, further research is needed.

\section{Neutrino Emission from an Astrophysical Source?}

Perhaps the most definitive result regarding the question of the constitution of Blazar jets would come with the detection of a neutrino flux from such a system.

On 22 September 2017, the ICECUBE Collaboration showed data from a neutrino of very high energy, indicating that the particle might have originated from an AGN. The neutrino detection alert was promptly disseminated to other observing teams, and the Fermi-LAT reported that the direction of the neutrino was in line with a known gamma-ray source which was in an active state: the blazar TS 0506+056. In addition, the MAGIC telescopes showed that a source of extremely high energies in the same region of the sky.

If these detections are corroborated, it strongly suggests that the contents of astrophysical jets from Blazars are hadronic. Clearly, more observations and modeling are necessary for this conclusion to be fully understood. Remarkably, the recent spectral observations of gold produced by the kilonova associated with gravitational waves detections from LIGO/VIRGO (see, e.g. Seigel, 2019) suggests that hadronic jets form even during compact object coalescence via gravitational wave generation, and that these jets can have significant hadronic components. 


\section{Concluding Remarks}

The data and models discussed herein suggest a model for the jet structures in which beams or blobs of energetic plasmas propagate outward from the jet central engine to interact with the ambient medium in the central regions of these sources. This ambient medium in many cases comes from prior ejecta from the central source, but can also come from clouds in the Broad Line Region. The jet can apparently also excavate large regions, as is suggested by the complex structures in, for example, 3C120. The physical processes which can accelerate and entrain the ambient medium through which the jet propagates have been discussed in detail in several venues (see, e.g., Rose et al.,1984, 1987, Beall, 1990, van der Westhuizen et al. 2019). It is also possible given analyses by Kundt and Gopal-Krishna (2004) and subsequent authors, that some of the jets can consist of electron-positron pairs. This thesis is especially intriguing for pulsar-driven jets. But perhaps most importantly it needs to be investigated in light of the line-signatures that show heavy elements in the recent kilonova associated with NS-NS star coalescence that produced gravity wave detections.

In this review, we have focused on historical developments of the investigations of astrophysical jets which shed light on their origin and evolution, a study which represents a series of "stations" of our evolving understanding.

The data can be interpreted as being associated with a jet-launch scenario for these sources, and suggests an association between a jet launch in the MOJAVE data for 3C454.3 and its $\gamma$-ray flare from the Fermi LAT. In addition, jets from microquasars and protostellar systems show similar patterns of variability to those of AGNs. These data require us to abandon our assumptions both of spherical symmetry and of single-zone productions in our models of these sources (Dar, 2009).

Clearly, the recent results of neutrino emission associated with a single Blazar bear further study, as does the continued work by observers of AGNs by the EHT and MOJAVE.

The author gratefully acknowledges the support of the Chief of Naval Research for these efforts. This research has made use of data from the MOJAVE database that is maintained by the MOJAVE team (Lister et al., 2009, AJ, 137, 3718). The author gratefully acknowledges the data provided by the MOJAVE Team for this paper.

\section{References}

[1] Fermi LAT Collaboration; Abdo, A. A.; Ackermann, M.; Ajello, M.; Axelsson, M.; Baldini, L.; Ballet, J.; Barbiellini, G.; Bastieri, D.; Baughman, B. M.; and 167 coauthors: 2009, Science, 326.1512F (Modulated High-Energy Gamma-Ray Emission from the Microquasar Cygnus X-3)

[2] Abdo, A. A.; Ackermann, M.; Ajello, M.; Atwood, W. B.; Axelsson, M.; Baldini, L.; Ballet, J.; Barbiellini, G.; Bastieri, D.; Baughman, B. M.; and 173 coauthors: 2009, Ap. J., 701L,123A (Fermi LAT Observations of LS I $+61^{\circ} 303$ : First Detection of an Orbital Modulation in $\mathrm{GeV}$ Gamma Rays)

[3] The Pierre Auger Collaboration, et al., 2007, Science 318, 938.

[4] Bach, U., Villata, M., Raiteri, C. M., et al. 2006, "Structure a variability in the VLBI jet of BL Lacertae during the WEBT campaigns (1995-2004)," A.A., 456, 105. 
[5] Beall, J.H. et al., 1978, Ap. J., 219, 836.

[6] Beall, J.H., Rose, W.K., 1980, Ap. J., 238, 579.

[7] Beall, J.H., 1987, Ap. J., 316, 227.

[8] Beall, J.H., 1990, Physical Processes in Hot Cosmic Plasmas (Kluwer: Dordrecht), W. Brinkmann, A. C. Fabian, and F. Giovannelli, eds., pp. 341-355.

[9] Bisnovatyi-Kogan, Gennady, Ardelyan, Nikolai, and Moiseenko, Sergei, 2002, Mem. S.A.It. 73, 1134., in Proceedings of the Vulcano Workshop on High-Energy Cosmic Sources, 2001

[10] Bisnovatyi-Kogan, Gennady, Klepnev, Alexandr S., and Lovelace, Richard V.E., 2013, Acta Polytechnica, 53 (Supplement), 667-682.

[11] Bonning, E. W., Bailyn, C., Urry, C. M., Buxton, M., Fossati, G., Maraschi, L., Coppi, P.,Scalzo, R., Isler, J., Kaptur, A. 2009, Astrophys. J. Lett., 697, L81.

[12] Bradshaw, C.F., Fomalont, E.B., and Geldzahler, B.J.,1999, The Astrophysical Journal. 512 (2): L121-L124. Bibcode:1999ApJ...512L.121B.

[13] Fomalont, E.B.; Geldzahler, B.J. and Bradshaw, C.F., 2001, The Astrophysical Journal, 558:283-301,

[14] Carilli, C.L. et al.: 1996, The A\&A Rev 7, 1-54.

[15] Chaty, S.: 1998, PhD Thesis (Multi-wavelength study of the microquasar GRS 1915+105 and of high-energy binary sources in the Galaxy)

[16] Chaty, S., 2007, in Frontier Objects in Astrophysics and Particle Physics, F. Giovannelli and G. Mannocchi (eds.), Italian Physical Society, Editrice Compositori, Bologna, Italy, 93, 329.

[17] Doelman, S.S. et al., "First M87 Event Horizon Telescope Results. III. Data Processing and Calibration.” ApJL, 875, L3, Pp. 1-32

[18] Dar, Arnon, 2009, private communication.

[19] Doeleman et al., 2019, at https : //iopscience.iop.org/journal/2041 8205/page/Focus n $_{E} H T$

[20] Dwahan, V., Mirabel, I.F., and Rodriguez, L.F., 2000, , 543:373-385.

[21] Doolin, S., and Blundell, K.M., 2009, Ap. J.,698, L23.

[22] Fish, V.L, et al., in Galaxies 2016, 4, 54; doi:10.3390/galaxies4040054 www.mdpi.com/journal/galaxies

[23] Fomalont, E., Geldzahler, B., Bradshaw, C., 2001, Ap. J. 558, 283-301.

[24] Giovannelli, F., Sabau-Graziati, L., 2004, Space Science Reviews, 112, 1-443 (Kluwer Academic Publishers: Netherlands). 
[25] Gómez et al. 2000, Science 289, 2317.

[26] Hiribayashi et al., 2000 PASJ 52, 997.

[27] Hannikainen, D.C., Rodriguez, J., 2008, in Multifrequency Behavior of High Energy Cosmic Sources, Chin. J. A\&A 8, Suppl., 341.

[28] Hawley, J.F., 2003, Phys. Plasmas 10, 1946-1953.

[29] IceCube CollaborationScience "Neutrino emission from the direction of the blazar TXS 0506+056 prior to the IceCube-170922A alert", 12 July 2018, Science, 147-151.

[30] Jorstad, S.G., Marscher, A.P., Lister, M.L., Stirling, A.M., Cawthorne, T.V. et al., 2005, AJ 130, 1418-1465.

[31] Jorstadt, S., Marscher, A., Stevens, J., Smith, P., Forster, J. et al., 2006, in Multifrequency Behavior of High Energy Cosmic Sources, Chin. J. A\&A 6, Suppl. 1, 247.

[32] Jorstad, Svetlana G., et al., 2013, Astrophysical Journal, 773, 147.

[33] Kundt, W. and Gopal-Krishna, 2004, Journal of Astrophysics and Astronomy, 25, 115-127.

[34] Krause, M., Camenzind, M., 2003, in The Physics of Relativistic Jets in the CHANDRA and XMM Era, New Astron. Rev. 47, 573.

[35] Lightman, A. P., Eardley, D. N., 1974, Ap. J. Letters 187, L1.

[36] Lister et al., 2009, A.J., 137, 3718.

[37] Madejski, Greg M., et al., 1999, ApJ, 521, 145-154.

[38] Marscher, A.P., et al., 2002, Nature, 417, 625-627.

[39] Marscher, A.P., 2006, in Multifrequency Behavior of High Energy Cosmic Sources, Chin. J. $A \& A$ 6, Suppl. 1, 262.

[40] Mioduszewski, A. J., Rupen, M. P., Walker, R. C., Schillemat, K. M., \& Taylor, G. B. 2004, BAAS, 36, 967.

[41] Mirabel, I. F.; Dhawan, V.; Chaty, S.; Rodriguez, L. F.; Marti, J.; Robinson, C. R.; Swank, J.; Geballe, T., 1998, A.\& A., 330L, 9M (Accretion instabilities and jet formation in GRS $1915+105)$.

[42] Mirabel, I. F.; Chaty, S.; Rodriguez, L. F.; Sauvage, M., 2015, "Jet-induced star formation by a microquasar", in Extragalactic jets from every angle, Proceedings of the International Astronomical Union, IAU Symposium, Volume 313, pp. 370-373.

[43] Mushotzky, R.F., Serlemitsos, P.J., Becker, R.H., Boldt, E.A., and Holt, S.S., Ap. J. 220, 790-797.

[44] ICECUBE COLLABORATION, Science 13 Jul H: Vol. 361, Issue 6398, pp. 147-151. 
[45] Paredes, J., 2007, in Frontier Objects in Astrophysics and Particle Physics, F. Giovannelli \& G. Mannocchi (eds.), Italian Physical Society, Editrice Compositori, Bologna, Italy, 93, 341.

[46] Perucho, M. and Bosch-Ramon, V., 2012, A\&A, Vol. 539, id. A57, 15 pp.

[47] Rahoui, F.; Chaty, S.; Rodriguez, J.; Fuchs, Y.; Mirabel, F., 2008, "Proceedings of the VII Microquasar Workshop: Microquasars and Beyond. September 1 - 5, 2008. Foca, Izmir, Turkey. Published online at http://pos.sissa.it/cgi-bin/reader/conf.cgi?confid=62, p.36" (Multiwavelength monitoring of the microquasar GRS 1915+105)

[48] Romanova, M. and Lovelace, R., 2005, 2009, Triggering of Relativistic Jets, (Instituto de Astronomia, Universidad Nacional Autonoma de Mexico, William H. Lee and Enrico RamirezRuiz, eds.); also at arXiv:0901.4753v1, astro-ph.HE, 29 Jan 2009.

[49] Rose, W.K., et al., 1984, Ap. J. 280, 550.

[50] Rose, W.K., et al., 1987, Ap. J. 314, 95.

[51] Silk, J., Antonuccio-Delogu, V, Y. Dubois, Y, Gaibler,V., Haas6, M.R. , S. Khochfar, S., \& Krause, M., A 545, L11 (2012)

[52] Seigel, D.M., 2019, https://arxiv.org/abs/1901.09044.

[53] Ulrich, Marie-Helene, Maraschi, Laura, and Urry, C. Megan, 1997, Ann. Rev. A\&A, 35, 445-502.

[54] Urry, C. Megan, and Padovani, Paolo, 1995, PASP 107, 803.

[55] Urry, C. Megan, 2011, J. Astrophys. Astr. 32, 139-145

[56] van der Laan, H., 1966, Nature 211, 1131.

[57] van der Westhuizen, I.P., van Soelen, B., Meintjes, P.J. and Beall, J.H., 2019, M.N.R.A.S., Vol. 485, Issue 4, June 2019, 4658-4666.

[58] Zanni, C., Murante, G., Bodo, G., Massaglia, S., Rossi, P., Ferrari, A., 2005, A\&A, 429, 399.

[59] Waisberg, I., Dexter, J., Olivier-Petrucci, P., Dubus, G., and Perraut, K., 2019, A\&A 624, A127

\section{DISCUSSION}

MARTIN TOPINKA: Could you comment on the origin of the infrared emission of the jets?

JIM BEALL: I belive the infrared emission from the jets arises based in heating of the jet and the ambient medium through turbulence. Of course, synchrotron emission can extend into the infrared also, and relativistic particle interactions can be a source of significant heating. But this is quite an interesting question, and deserves further study. 
DIMITRI BISIKALO: Can you kindly comment on the behavior of the accretion disk in the slide with the radio jet?

JIM BEALL: In the example from the Owens Valley data, the polarization of the millimeter wavelength observations suggests that the jet is coming off in a roughly perpendicular direction from the plane of the accretion disk. This will of course make sense if the disk is generating the jet through instabilities in its inner region. But the actual mechanism that can produce this deserves further study.

MARTIN TOPINKA: Why is the jet flow so collimated and without too much turbulence between the soure input and the "main" head of the jet?

JIM BEALL: I think that the highly collimated nature of the jet can only be explained by an extended and energetically significant magnetic field that provides a cocoon for the jet to propagate through. That said, it is also possible that the jet itself can generate such a magnetic field within itself by net currents in its rest frame as the energy losses from light particles (electrons and positrons) cause them to slow, while the hadronic particles continue to propagate with relatively fewer losses. Of course,eventually the turbulence generated by the non-linear interactions of the jet and the ambient medium (and the jet with itself) produces the shock structures at the head of the jet. 